\title{
Estudio de la actividad antioxidante, antimicrobiana y toxicidad de tres extractos de Heliocarpus appendiculatus Turcz (malvaceae)
}

\author{
Antioxidant, antimicrobiant, and toxicity activity of three extracts of Heliocarpus appendiculatus Turcz \\ (malvaceae) \\ Alejandro Hernández-López ${ }^{*}$, Mayra Cruz-Hernández¹, Diana Guerra-Ramírez²; \\ José Maria Cunill ${ }^{3}$, Priscila Guerra-Ramírez², Fernando Rivera-Cabrera ${ }^{4}$, \\ Oscar Abelardo Ramírez-Marroquin ${ }^{1}$, Alma Xochil Avila-Alejandre ${ }^{1}$
}

\begin{abstract}
RESUMEN
Heliocarpus appendiculatus Turcz (jonote) es usado en la medicina tradicional en el centro, sur y sureste de México; sin embargo, la información científica de sus actividades biológicas específicas es escasa. El objetivo de este trabajo fue evaluar la actividad antioxidante, antimicrobiana y la toxicidad de extractos orgánicos de diferente polaridad obtenidos a partir de hojas de esta planta. El extracto metanólico presentó la mayor actividad antioxidante $(1251 \pm 44 \mu \mathrm{M}$ de TEAC para ABTS y $565 \pm 51 \mu \mathrm{M}$ de TEAC para DPPH), así como el mayor contenido de fenoles totales (48.3 mgEAg/ge). El extracto obtenido con cloruro de metileno mostró actividad antimicrobiana sobre E. coli $(p<0.05)$. Este mismo extracto presentó la mayor actividad tóxica en Artemia franciscana, el cual disminuyó la viabilidad hasta $70 \%$ y presentó una concentración mínima tóxica de 1 ppm $(p<0.05)$.
\end{abstract}

PALABRAS CLAVE

Jonote, nauplis, actividad biológica, hojas

\section{ABSTRACT}

Heliocarpus appendiculatus Turcz (jonote) is used in traditional medicine in central, southern and southeastern Mexico; however, scientific information on their specific biological activities is scarce. The objective of this work was to evaluate the antioxidant, antimicrobial and toxicity activity of organic extracts of different polarity, obtained from the leaves of this plant. The methanolic extract showed the highest antioxidant activity (1251 $\pm 44 \mu \mathrm{M}$ TEAC for ABTS and $565 \pm 51 \mu \mathrm{M}$ TEAC for DPPH) and the highest total phenolic content (48.3 mgEAg/ge). The extract obtained with methylene chloride showed antimicrobial activity on E. coli $(p<0.05)$. This same extract showed the highest toxic activity in Artemia franciscana, which decreased viability up to $70 \%$ and presented a minimum toxic concentration of $1 \mathrm{ppm}(p<0.05)$.

KEYWORDS

Jonote, nauplis, biological activity, leaf

\footnotetext{
${ }^{1}$ Instituto de Biotecnología, Campus Tuxtepec, Universidad del Papaloapan, Circuito Central \# 200, Col. Parque Industrial, San Juan Bautista Tuxtepec. 68300, Oaxaca, México.

${ }^{2}$ Laboratorio de Productos Naturales, Área de Química, Departamento de Preparatoria Agrícola, Universidad Autónoma Chapingo, carretera México-Texcoco km 38.5. 56230, Chapingo, Estado de México, México.

${ }^{3}$ Departamento de Biotecnología, Universidad Politécnica Metropolitana de Puebla, calle Popocatepetl s/n, Col. Tres Cerritos, Puebla. 72480, Puebla, México.

${ }^{4}$ Laboratorio de Postcosecha de Recursos Fitogenéticos y Productos Naturales, Departamento de Ciencias de la Salud, Universidad Autónoma Metropolitana, Av. San Rafael Atlixco 186, Col. La Purisima, Iztapalapa. 09340, Ciudad de México, México.
}

*Autor para correspondencia: alehz74@yahoo.com.mx

Fecha de recepción: 16 de diciembre de 2018

Fecha de aceptación: 29 de agosto de 2019

Disponible en línea: 25 de noviembre de 2019 


\section{INTRODUCCIón}

Heliocarpus es un género distribuido desde México hasta Centro, Sudamérica y el Caribe (Standley y Steyermark 1949); comprende un amplio grupo de especies, conocidas de manera general como "jonotes". Antiguamente clasificados dentro de la familia Tiliaceae, recientemente se han reclasificado dentro de Malvaceae (Stevens 2001). Heliocarpus appendiculatus Turcz es conocido con diferentes nombres coloquiales, tales como: jonote, calagua, mozote, palo baboso, etc. Es un árbol presente en selvas altas y medianas perennifolias y subperennifolias; este árbol comúnmente se desarrolla en sitios que han sido perturbados por actividades antropogénicas, por lo que se le considera como especie pionera (Cunill-Flores comunicación personal).

Su área de distribución va desde la zona de las Huastecas, en San Luis Potosí, y el norte de Puebla, hasta el sur de Veracruz, en la zona de los Tuxtlas, y del norte de Oaxaca hasta el norte de Chiapas (Pennington y Sarukhán 2005; Vibrans 2009). H. appendiculatus ha sido descrito como un árbol de hasta $15 \mathrm{~m}$, con hojas de entre 14 y $16 \mathrm{~cm}$ de largo, por entre 12 y $14 \mathrm{~cm}$ de ancho, ovaladas, a veces trilobadas, glabras por el haz y tomentosas por el envés; con dos apéndices foliares en la parte basal de la lámina (Macía 1999).

En cuanto a sus usos tradicionales, Frei et al. (1998) y Leonti (2018) describieron que, en la Sierra de Oaxaca, la corteza y la savia son usadas para el tratamiento de enfermedades dermatológicas, así como otras relacionadas con el sistema musculoesquelético. Sin embargo, extractos etanólicos de este árbol colectados en Costa Rica fueron probados frente a diversas especies de hongos que afectan la piel y mucosas, sin mostrar actividad antifúngica (Svetaz et al. 2010).

En otro estudio, se evaluó la actividad antimalárica del extracto etanólico de la corteza de H. appendiculatus, y la reportaron como activa-débil (Chinchilla-Carmona et al. 2004). Adicionalmente, el extracto hidroalcohólico muestra una actividad promisoria anti-Leishmania. Por otro lado, en un sondeo etnofarmacobiológico, efectuado en la región central de Perú, se colectaron 249 especies de plantas con usos medicinales, entre ellas Heliocarpus americanus L.; los usos encontrados para esta especie son los siguientes: la corteza se utiliza para aliviar el dolor de estómago; las hojas calientes, en forma de cataplasma, alivian el dolor de riñón, el dolor producido por los golpes, los hematomas (moretones) y la hinchazón; un líquido pegajoso obtenido al hervir la corteza del tronco, junto con algunos brotes de los ápices, se ingiere todas las mañanas durante los últimos días del embarazo para ayudar al alumbramiento (Valadeau et al. 2010).

En México, la corteza del árbol de jonote se usa comúnmente en la elaboración de artesanías y de papel amate, los cuales son una fuente de ingresos para los campesinos de la región (Patiño 2018). Cunill (2015) registró que, en la comunidad totonaca de Ecatlán, Puebla, México, la madera de $H$. appendiculatus se usa en la construcción de casas, chiqueros, corrales, etc.; en cuanto a su uso medicinal, es utilizado para el tratamiento del asma, así como para aliviar los moretones y la inflamación resultantes de golpes; asimismo, con las hojas se preparan infusiones y cataplasmas utilizados para acelerar el parto.

El jonote tiene un valor terapéutico empírico interesante. Sin embargo, los reportes científicos acerca de su actividad biológica son escasos. Por lo tanto, en el presente trabajo se llevó a cabo la evaluación de la actividad antioxidante, antimicrobiana y toxicológica de extractos orgánicos de diferente polaridad obtenidos de hojas de $H$. appendiculatus.

\section{Material Y MÉtodos}

El material biológico se colectó en el municipio de Jonotla, ubicado en la Sierra Nororiental del estado de Puebla (20을 $01^{\prime} 24^{\prime \prime}$ y $20^{\circ} 09^{\prime} 12^{\prime \prime}$ de latitud norte y 97을 26' 54" y 97으 36' 00" de longitud occidental) (INEGI 2015). Un ejemplar se identificó y fue depositado en el Herbario y Hortorio Jorge Espinosa Salas "JES", del Área de Biología, del Departamento de Preparatoria Agrícola, de la Universidad Autónoma Chapingo, con el número de registro 25498. Se obtuvieron tres extractos de $H$. appendiculatus, con solventes de diferente polaridad, usando una proporción $4 / 1 \mathrm{v} / \mathrm{v}$ $(2.4 \mathrm{~kg} / 5 \mathrm{~L}$ de solvente $\mathrm{p} / \mathrm{v})$.

Las hojas secas y molidas se maceraron con hexano $\left(\mathrm{C}_{6} \mathrm{H}_{14}\right)$, a temperatura ambiente $\left(26 \pm 2{ }^{\circ} \mathrm{C}\right)$, durante $48 \mathrm{~h}$; posteriormente, se filtraron y se retiraron las grasas precipitadas. El residuo vegetal fue sometido a dos extracciones más con hexano; en cada ocasión, el disolvente se evaporó en un Rotavapor ${ }^{\circledR}$ R-210 (BÜCHI Labortechnik, Alemania), que cuenta con un baño calefactor B-491® (BÜCHI Labortechnik, Alemania) y se reservó el extracto. El residuo vegetal fue sometido a extracción con cloruro de metileno y con metanol, repitiendo el mismo procedimiento antes descrito. Finalmente, se calculó el rendimiento de sólidos totales obtenidos con cada disolvente, promediando el peso del extracto obtenido en cada maceración. 


\section{Preparación de las soluciones de trabajo de extractos de H. appendiculatus}

Una cantidad adecuada de sólidos de cada uno de los extractos se dispersó en agua destilada con Tween ${ }^{\circledR}$ 80 (Sigma-Aldrich, EUA) ${ }^{1}$ a $0.2 \%$ p/v, con ayuda de una pipeta automática, para obtener una solución madre con $10 \mathrm{mg} / \mathrm{mL}$. La disolución se mantuvo en refrigeración y protegida de la luz hasta su uso (Guerra-Ramírez et al. 2019).

\section{Determinación de la actividad antioxidante}

La actividad antioxidante se determinó mediante dos técnicas: el ensayo de secuestro del radical difenil-2-picril-hidrazol (DPPH) y el ensayo de decoloración del radical 2,2'-azino-di (3-etilbenzotiazolina-6-ácido-sulfónico) (ABTS•+). En ambos casos, para evaluar la actividad antioxidante, se preparó una curva estándar con $0,0.5,1.0,1.5,2.0$ y $2.5 \mathrm{mM}$ de Trolox ${ }^{\circledR}$ (Sigma Aldrich, EUA), a partir de una solución madre $4 \mathrm{mM}$, disuelta en Buffer salino de fosfatos (PBS) a $\mathrm{pH}$ $7.4\left(10 \mathrm{mM} \mathrm{Na}_{2} \mathrm{HPO}_{4}, 2 \mathrm{mM} \mathrm{KH} \mathrm{PO}_{4}, 140 \mathrm{mM} \mathrm{NaCl}\right.$, $2 \mathrm{mM} \mathrm{KCl}$ ). El ensayo de DPPH se efectuó de acuerdo con Sharma y Bath (2009), con algunas modificaciones que se describen a continuación (Roldán-Sabino et al. 2018; Zou et al. 2014): la reacción se realizó en un volumen final de $1 \mathrm{~mL}$ y se usó metanol como disolvente. Se tomaron $800 \mu \mathrm{L}$ de una solución $50 \mu \mathrm{M}$ de DPPH en metanol, se mezclaron con $200 \mu \mathrm{L}$ de las soluciones madre de cada extracto o las diferentes concentraciones de Trolox® y se incubaron por $30 \mathrm{~min}$ en oscuridad. Se midió la absorbancia a $517 \mathrm{~nm}$ en un espectrofotómetro BIOTRAZA® 722 (Biotraza, China). La probable actividad antioxidante del Tween ${ }^{\circledR} 80$ fue descartada, evaluando diferentes concentraciones de Tween ${ }^{\circledR} 80(0.1,2,3$ y 5\%), con DPPH, como se describió previamente y monitoreando los cambios en la absorbancia cada 5 min durante $30 \mathrm{~min}$.

El ensayo de decoloración del radical 2,2'-azino-di (3-etilbenzotiazolina-6-ácido-sulfónico)(ABTS•+), se llevó a cabo de acuerdo con Mendoza-Jiménez et al. (2018) y Re et al. (1999). Para la formación del radical $\mathrm{ABTS} \bullet+$, se mezclaron $10 \mathrm{~mL}$ ABTS $(2 \mathrm{mM})$, previamente disuelto en PBS a $\mathrm{pH} 7.4$ (preparado como se mencionó arriba), con $40 \mu \mathrm{L}$ de Persulfato de potasio $70 \mathrm{mM}$. La mezcla se incubó a temperatura ambiente $\left(26 \pm 1^{\circ} \mathrm{C}\right)$, en oscuridad, por $16 \mathrm{~h}$. Para efectuar el ensayo, se ajustó el espectro a $734 \mathrm{~nm}$ y se midió la absorbancia del ABTS•+; se diluyó con PBS

\footnotetext{
${ }^{1}$ Aditivo alimentario usado para emulsionar grasas y como agente dispersante en fármacos.
}

a pH 7.4 hasta llegar a una absorbancia $\left(\mathrm{Abs}_{734 \mathrm{~nm}}\right) \mathrm{de}$ $0.700 \pm 0.030$. Posteriormente, se mezclaron $990 \mu \mathrm{L}$ de esta solución ajustada, con $10 \mu \mathrm{L}$ de solución madre $(10 \mathrm{mg} / \mathrm{mL})$ de cada extracto. La mezcla se incubó en la oscuridad por $6 \mathrm{~min}$, e inmediatamente se registró la absorbancia a la longitud de onda antes mencionada. Para ambos ensayos, los resultados se expresaron como capacidad antioxidante equivalente a Trolox ${ }^{\circledR}$ (TEAC, por sus siglas en inglés), empleando los valores de la curva patrón de Trolox® respectiva, de la siguiente manera: primero, se calculó el porcentaje de inhibición (\%I) mediante la siguiente fórmula:

$$
\% \mathrm{I}=[(\mathrm{AB}-\mathrm{AA}) / \mathrm{AB}] \times 100
$$

Donde:

$\mathrm{AB}=$ la absorbancia del blanco a $517 \mathrm{~nm}$ para $\mathrm{DPPH}$ o a $734 \mathrm{~nm}$ para ABTS

$\mathrm{AA}=$ la absorbancia de la muestra con antioxidante a $517 \mathrm{~nm}$ para DPPH o a $734 \mathrm{~nm}$ para ABTS

Posteriormente, con los valores, se generó una gráfica de \% $\mathrm{I}_{\mathrm{DPPH}} v s$ Concentración de Trolox ${ }^{\circledR}$ y se calculó la concentración de $\mu \mathrm{M}$ TEAC usando la siguiente fórmula:

$$
\mu \mathrm{M} \text { TEAC }=\left(\% \mathrm{I}_{\mathrm{DPPH}}-\mathrm{b}\right) / \mathrm{m}
$$

Donde:

$\% \mathrm{I}_{\mathrm{DPPH}}=$ porcentaje de inhibición de la muestra

$\mathrm{b}=$ la intersección

$\mathrm{m}=$ la pendiente

Finalmente, los valores de TEAC para DPPH y ABTS se corrigieron con la cantidad de extracto correspondiente y se reportaron como $\mu \mathrm{M}$ de TEAC/g extracto.

El contenido de fenoles totales se determinó por el método de Folin-Ciocalteu de la siguiente manera: a $0.2 \mathrm{~mL}$ de la solución madre de extracto se le agregó $1 \mathrm{~mL}$ de reactivo de Folin-Ciocalteu ${ }^{\circledR}$ (MEYER, México) a $10 \%$ (v/v) y $0.8 \mathrm{~mL}$ de $\mathrm{Na}_{2} \mathrm{CO}_{3}$ a $20 \%$ $(\mathrm{p} / \mathrm{v})$. La mezcla se incubó a temperatura ambiente durante $2 \mathrm{~h}$. Se midió la absorbancia de la mezcla de reacción de los extractos a $760 \mathrm{~nm}$. Los resultados se interpolaron en una curva de calibración de ácido 
gálico que se preparó a partir de una solución madre $0.1 \mathrm{mg} / \mathrm{mL}$. Las mediciones se hicieron por triplicado y los resultados del contenido de fenoles totales se expresaron en miligramos de fenoles equivalentes a ácido gálico por gramos de extracto (mg EAG/ge). Adicionalmente, se llevó a cabo una determinación cualitativa de alcaloides, para lo cual se pesaron 10 $\mathrm{mg}$ de cada extracto y se disolvieron en $2 \mathrm{~mL}$ de etanol; se filtró $1 \mathrm{~mL}$ de cada uno; se agregaron $10 \mu \mathrm{L}$ de $\mathrm{HCl} 1 \mathrm{~N}$, y se agregó, gota a gota, el reactivo de Mayer, hasta la aparición de un precipitado blanco.

\section{Actividad antimicrobiana}

Para probar la actividad antimicrobiana se emplearon dos bacterias consideradas no patógenas, una representativa del grupo Gram - (Escherichia coli) y otra de las Gram + (Bacillus thuringiensis). La actividad antimicrobiana se llevó a cabo por el método de Kirby-Bauer (Bauer et al. 1996), con algunas modificaciones: los sensi-discos se prepararon usando $140 \mu \mathrm{g}$ de cada extracto, se usó gentamicina $(100 \mu \mathrm{g} /$ $\mathrm{mL}$ ) como control positivo y Tween ${ }^{\circledR} 80$ al $3 \%$ como control negativo (-). Las cajas Petri con $20 \mathrm{~mL}$ de Luria- Bertani o Medio Mueller-Hinton se inocularon con $100 \mu \mathrm{L}$ de (Gram -) o B. thuringiensis (Gram +), respectivamente. Las cajas se incubaron por $24 \mathrm{~h}$ a 37 $\pm 0.5{ }^{\circ} \mathrm{C}$. El ensayo se hizo por triplicado. Cumplido el tiempo de incubación, las cajas Petri fueron fotodocumentadas en un escáner Brother ${ }^{\circledR}$ (Brother Corp, China) modelo MFC-J475DW, con una resolución de 1 200 ppp y se midieron los halos de inhibición usando el software Image J ${ }^{\circledR}$ (Rasband, 1997). Los resultados se reportaron como diámetro de inhibición relativo al control (-). La probable actividad antimicrobiana del Tween ${ }^{\circledR} 80$ fue descartada, incubando a $37^{\circ} \mathrm{C}, 5$ $\mathrm{mL}$ de medio Laura-Bertiani inoculado con $100 \mu \mathrm{L}$ de un cultivo fresco de E. coli o B. thuringiensis y monitoreando los cambios en la absorbancia a $600 \mathrm{~nm}$ cada hora durante $4 \mathrm{~h}$.

\section{Ensayos de toxicidad en Artemia franciscana}

Para los ensayos de toxicidad se emplearon nauplis de A. franciscana en estadio I; para ello, se eclosionaron quistes de procedencia comercial, incubándolos en solución salina + bicarbonato a $3.5 \% \mathrm{p} / \mathrm{v}$ a temperatura ambiente por $24 \mathrm{~h}$ con iluminación constante.

Para la exposición a los diferentes extractos, Tween ${ }^{\circledR} 80$ oSDS, se siguió la metodología reportada previamente por González et al. (2003), con algunas modificaciones (Guerra-Ramírez et al. 2019). Se usaron cajas de cultivo de 24 pocillos, preparadas de la siguiente manera: en cada pozo, se agregó el volumen necesario de la solución salina+bicarbonato a $3.5 \% \mathrm{p} / \mathrm{v}$ para ajustar las concentraciones de extracto $(0,1,5,10,50$ y 100 ppm), de Tween $\AA 80(0,0.5,1,2$ y $3 \%)$ o de SDS $(0,1,5,10,50$ y $100 \mathrm{ppm}$ ) a un volumen final de $2 \mathrm{~mL}$. En la primera columna de la caja de cultivo sólo se agregó salinatbicarbonato a $3.5 \% \mathrm{p} / \mathrm{v}$ (testigo de viabilidad). En las columnas subsecuentes se agregaron las diferentes concentraciones en orden creciente. Una vez ajustados los pocillos, se adicionaron 20 nauplis por pocillo. Las cajas de cultivo se incubaron a $22{ }^{\circ} \mathrm{C}$ durante 24 h. Finalizado el tiempo de exposición, se determinó la viabilidad con el siguiente criterio: los nauplis que se mantuvieron inmóviles por $10 \mathrm{~s}$ mientras estaban expuestos a luz constante se consideraron muertos. Cada concentración se ensayó usando 4 repeticiones. El efecto del Tween ${ }^{\circledR} 80$ fue descartado en un cultivo sumergido previo, usando $0,0.5,1,2$ y $3 \%$ de Tween ${ }^{\circledR}$ 80. Como control positivo se utilizó SDS.

\section{Análisis estadístico}

Para la actividad antioxidante, antimicrobiana y toxicológica, se utilizó un diseño experimental completamente al azar. Para la actividad antioxidante y antimicrobiana en cada ensayo se llevaron a cabo tres réplicas independientes, con tres repeticiones cada una (nueve determinaciones por cada extracto). Para la actividad toxicológica se hicieron tres réplicas, cada una con cuatro repeticiones. Para la estadística descriptiva se calcularon el promedio y la desviación estándar, y con esos datos se construyeron las gráficas de actividad antimicrobiana y toxicológica; para la actividad antioxidante, adicionalmente se calculó el coeficiente de variación. Se efectuó un análisis de variancia de una vía y para detectar diferencias entre los tratamientos se llevó a cabo el análisis de Tukey, con un $p<0.05$. Todos los análisis estadísticos se hicieron en el software MINITAB ${ }^{\circledR}$ ver 17 (State College, Pennsylvania, EUA).

\section{Resultados y discusión}

A partir de las hojas de $H$. appendiculatus se obtuvieron tres extractos; el de mayor rendimiento de sólidos totales fue el de cloruro de metileno con 51.1 g, seguido del metanólico con $46.1 \mathrm{~g} \mathrm{y}$, finalmente, el hexánico con 9.2 g. Estos datos sugieren que en esta planta predominan las sustancias de polaridad media y alta. Los extractos resultantes presentaron consistencia gomosa poco soluble en agua; por ello, 
para efectuar las disoluciones se utilizó Tween ${ }^{\circledR} 80$. Con la finalidad de determinar el efecto de Tween ${ }^{\circledR}$ 80 en los ensayos, se hicieron las pruebas en presencia de varias concentraciones de éste (figura 1a), donde se observó que el incremento en la cantidad de Tween ${ }^{\circledR}$ 80 no indujo cambios significativos $(\mathrm{P}>0.05)$ en la absorbancia del DPPH, durante los $30 \mathrm{~min}$ del ensayo, lo que demuestra que el Tween ${ }^{\circledR} 80$ no contribuye con
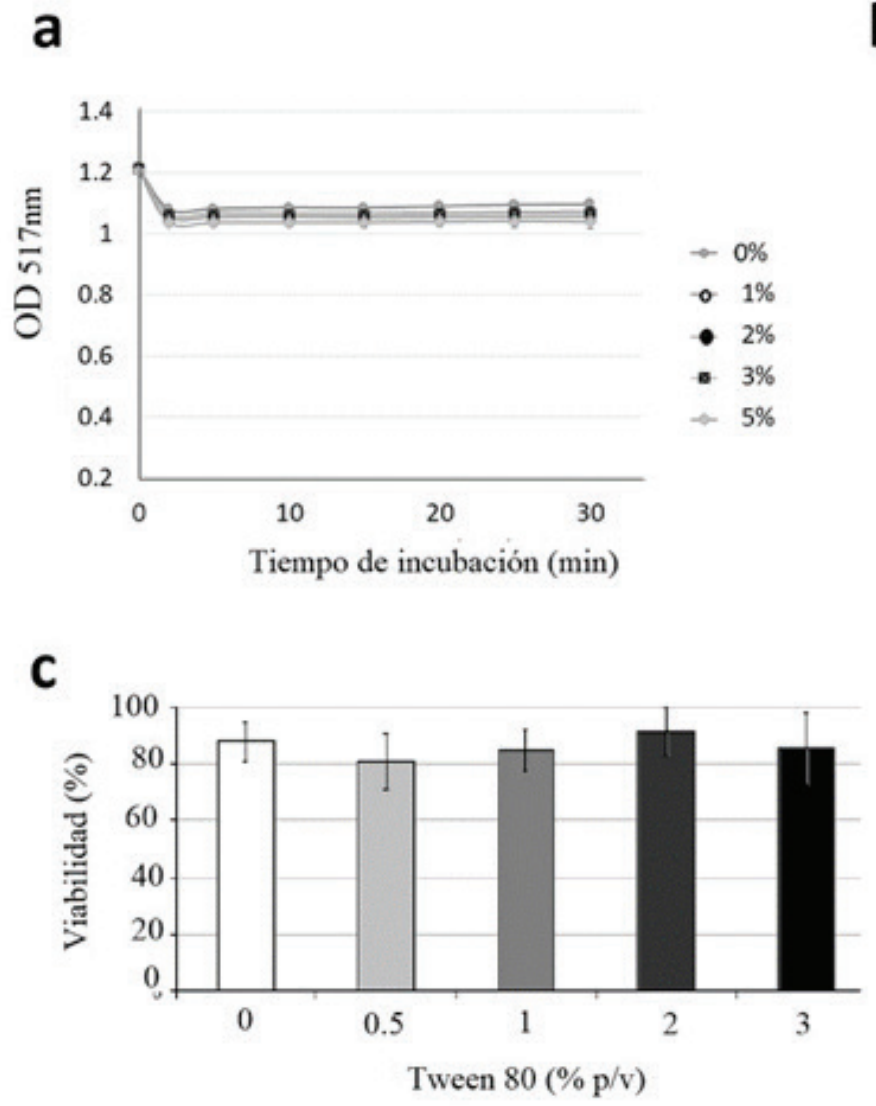

la actividad de secuestro del radical. Asimismo, las distintas concentraciones de Tween 80 no mostraron diferencias significativas $(\mathrm{P}>0.05)$ en cinética de crecimiento de E. coli (figura 1b). Finalmente, no se detectaron diferencias significativas $(\mathrm{P}>0.05)$ entre las distintas concentraciones evaluadas de Tween ${ }^{\circledR} 80$ sobre el porcentaje de viabilidad de nauplis (figura 1c).

b

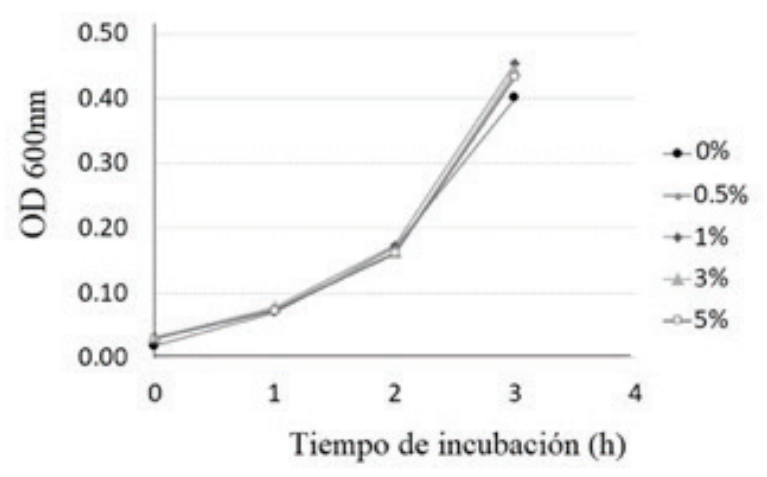

Tiempo de incubación (h)

Figura 1. Comportamiento de Tween ${ }^{\circledR} 80$ en los ensayos biológicos. a) Actividad antioxidante DPPH; b) Actividad antimicrobiana; c) Toxicidad.

Ninguno de los extractos logró inhibir 50\% del radical DPPH, por lo que no se pudo calcular la concentración inhibitoria 50 de DPPH (IC50 (cuadro 1). El extracto metanólico alcanzó los valores mayores de $\% \mathrm{I}_{\text {DPPH }}(34.3 \pm 2.8)$ y de TEAC en ambas técnicas: $565.3 \pm 51.2$ y $1241 \pm 45.1 \mu \mathrm{M}$ TEAC/g extracto, para DPPH y ABTS, respectivamente (cuadro 1). En el extracto de cloruro de metileno, se alcanzó aproximadamente la mitad de la actividad antioxidante del extracto metanólico, el $\% \mathrm{I}_{\mathrm{DPPH}}$ fue de $17 \pm 1.5$ y los valores de TEAC/g de extracto fueron de $260 \pm 26.7$ y $634 \pm 12$ para DPPH y ABTS, respectivamente (cuadro 1).
Finalmente, el extracto hexánico mostró los valores menores de actividad antioxidante en todas las técnicas. Los valores de \% $\mathrm{I}_{\mathrm{DPPH}}$ obtenidos en este trabajo, incluso en el extracto metanólico, muestran valores de capacidad antioxidante similares a los reportados para otras plantas, consideradas como fuentes potenciales de sustancias con propiedades antioxidantes, entre ellas los extractos de hoja de mesquite (Prosopis velutina Wooton), donde se reporta un $\% \mathrm{I}_{\text {DPPH }}$ de $34 \%$ (Ramírez-Rojo et al. 2019) y para jamaica (Hibiscus sabdariffa L.) se reportan valores de 1598 equivalentes de Trolox (Ruelas-Hernández et al. 2014). El análisis del contenido de fenoles 
totales muestra tendencia similar, ya que el extracto metanólico tiene la concentración mayor de fenoles con $48.3 \mathrm{mg}$ EAG $/ \mathrm{g}_{\mathrm{e}}$, seguido del extracto de cloruro de metileno, donde el contenido de fenoles fue de $14.9 \pm .7 \mathrm{mgEAG} / \mathrm{g}_{\mathrm{e}}$, y en el extracto hexánico sólo se cuantificaron $3.3 \mathrm{mgEAG} / \mathrm{g}_{\mathrm{e}}$ (cuadro 1). El contenido de polifenoles totales del extracto metanólico del jonote es mayor al reportado para el mismo tipo de extracto de hojas de cacao (30 mgEAG/g) (Quiñonez-Gálvez et al., 2013), de plantas jóvenes de moringa (10-12 mgEAG/g) (Cabrera-Carrión et al. 2017) y amaranto (32.3mgEAG/g) (Kraujalis et al. 2013) o extractos hidroalcohólicos de frutas como la piña, la guayaba y la guanábana, que presentan 8.9, 56.9, 39.6 mg EAG/100 g de fruta fresca, respectivamente (Jorge y Troncoso 2016).

Cuadro 1. Actividad antioxidante de extractos de Heliocarpus appendiculatus. Se comparan las diferencias de los valores de DPPH, ABST y Fenoles totales entre los extractos.

\begin{tabular}{|c|c|c|c|c|c|c|c|c|c|}
\hline \multirow[t]{2}{*}{\begin{tabular}{|c|} 
Extracto de \\
Jonote \\
\end{tabular}} & \multicolumn{3}{|c|}{ DPPH } & \multicolumn{3}{|c|}{ ABTS } & \multicolumn{3}{|c|}{ POLIFENOLES TOTALES $* * *$} \\
\hline & Promedio & $\mathrm{DE}$ & $\mathrm{CV}(\%)$ & Promedio & $\mathrm{DE}$ & $\mathrm{CV}(\%)$ & Promedio & $\mathrm{DE}$ & $\mathrm{CV}(\%)$ \\
\hline \multicolumn{10}{|l|}{ Metánolico } \\
\hline$\% \mathrm{I} *$ & $34.2^{\mathrm{a}}$ & \pm 2.8 & 8.4 & $65.7^{\mathrm{a}}$ & \pm 2.2 & 3.4 & \multirow[b]{2}{*}{$48.3^{\mathrm{a}}$} & \multirow[b]{2}{*}{ \pm 0.6} & \multirow[b]{2}{*}{1.3} \\
\hline TEAC $* *$ & $565.3^{\mathrm{a}}$ & \pm 51.2 & 9.1 & $1251^{\mathrm{a}}$ & \pm 45.1 & 5.8 & & & \\
\hline \multicolumn{10}{|l|}{ Hexánico } \\
\hline$\% \mathrm{I}$ & $14.0^{\mathrm{b}}$ & \pm 0.98 & 7.0 & $15.3^{\mathrm{b}}$ & \pm 1.6 & 10.54 & \multirow[b]{2}{*}{$3.3^{\mathrm{c}}$} & \multirow[b]{2}{*}{ \pm 0.2} & \multirow[b]{2}{*}{8.1} \\
\hline TEAC & $206.2^{\mathrm{b}}$ & \pm 17.4 & 8.4 & $219^{b}$ & \pm 26 & 11.92 & & & \\
\hline \multicolumn{10}{|c|}{ Cloruro de metileno } \\
\hline$\% \mathrm{I}$ & $17.0^{\mathrm{b}}$ & \pm 1.5 & 8.9 & $34.1^{\mathrm{c}}$ & \pm 2.9 & 8.8 & \multirow{2}{*}{$14.9^{\mathrm{b}}$} & \multirow{2}{*}{ \pm 0.7} & \multirow{2}{*}{4.90} \\
\hline TEAC & $260^{\mathrm{b}}$ & \pm 26.7 & 10.3 & $634^{\mathrm{c}}$ & \pm 12 & 7.1 & & & \\
\hline
\end{tabular}

Letras diferentes en el sentido de las columnas indican diferencias significativas de acuerdo con la prueba de Tukey ( $\mathrm{P}=0.05)$. DPPH= difenil-2-picril-hidrazol, ABTS=2,2'-azino-di (3-etilbenzotiazolina-6-ácido-sulfónico), DE= Desviación estándar, $\mathrm{CV}(\%)=$ Coeficiente de variación, *: \% $\mathrm{I}=$ Porcentaje de inhibición después de $30 \mathrm{~min},{ }^{* *}: \mathrm{TEAC}=$ Capacidad antioxidante equivalente a Trolox expresado en $\mu \mathrm{M} / \mathrm{g}$ Extracto, ${ }^{* * *}$ : Miligramos de fenoles equivalentes a ácido gálico por gramos de extracto (mg EAG/ge).

El método de difusión en agar mostró actividad antimicrobiana únicamente en el extracto de cloruro de metileno frente a E. coli (figura 2). La actividad antimicrobiana de extractos vegetales ha sido asociada con el contenido de fenoles, flavonoides y con otros compuestos no fenólicos, como los alcaloides (Escalona-Arranz et al. 2018); en el presente estudio, el extracto metanólico mostró el mayor contenido de fenoles; sin embargo, no se observó actividad antimicrobiana. En cambio, el extracto de cloruro de metileno con contenido de fenoles totales de sólo $14.9 \mathrm{mg}$ EAG $/ \mathrm{g}_{\mathrm{e}}$ mostró inhibición del crecimiento en E. coli. Una prueba cualitativa con el reactivo de Mayer mostró presencia de alcaloides en el extracto de jonote obtenido con dicho solvente (figura 2c), por lo que podrían ser estos compuestos los que participen en la inhibición microbiana. El extracto hexánico mostró una mayor cantidad de alcaloides en el ensayo cualitativo del reactivo de Mayer, aunque no actividad antimicrobiana; eso puede deberse a que el tipo de alcaloide con actividad antimicrobiana no se encuentra en este extracto o a que se requiere una mayor concentración. 
a)

b)

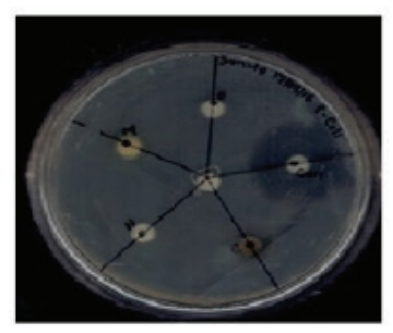

E. coli

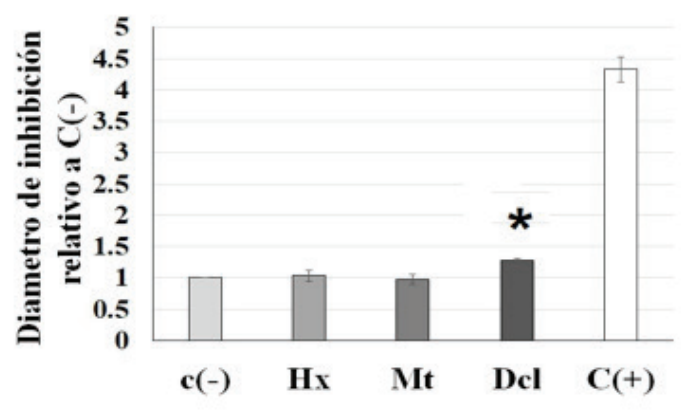

c)

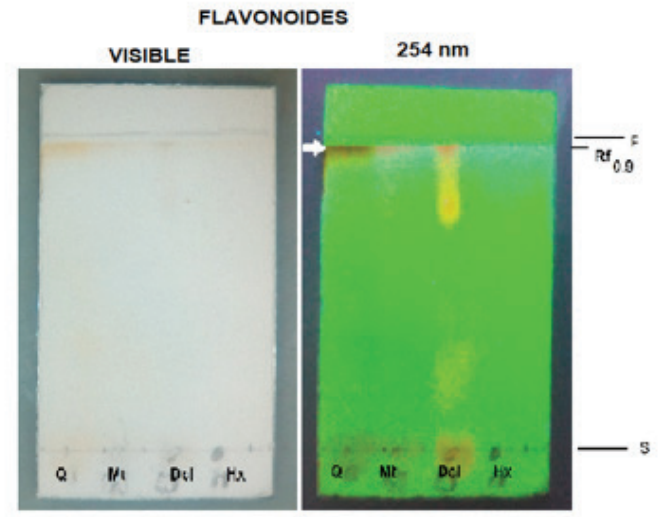

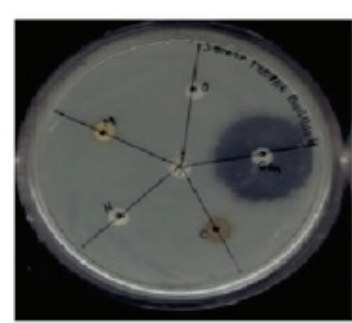

B. thuringensis

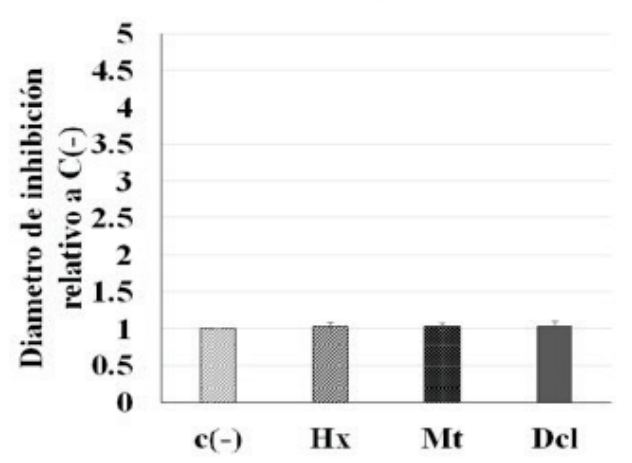

ALCALOIDES

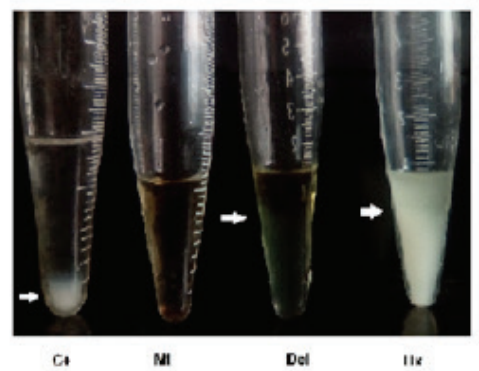

Figura 2. Actividad antimicrobiana de extractos de H. appendiculatus. a) antibiograma representativo; b) efecto de los extractos de jonote sobre la inhibición del crecimiento de E. coli y B. thuringiensis, comparados con el control (+); c) determinación

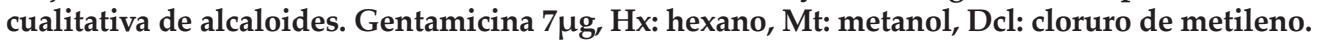

Finalmente, los ensayos de toxicidad en $A$. salina de los extractos de jonote mostraron que en el extracto metanólico no se encontraron diferencias estadísticamente significativas $(\mathrm{P}>0.05)$ en ninguna de las concentraciones probadas (figura 3). El promedio de viabilidad en los controles, sin la adición de ningún extracto, se mantuvo alrededor de 90\%, mientras que, con la adición de las diferentes concentraciones, el promedio se mantuvo por encima de $80 \%$ con una variabilidad de $18 \%$ (figura 3 ).

En el caso del extracto obtenido con cloruro de metileno (figura 3), se encontraron diferencias estadísticamente significativas en todas las con- centraciones probadas $(p<0.05)$ en comparación con el control. Sin embargo, con ninguna de las concentraciones probadas de los extractos se alcanzó una viabilidad menor o igual a $50 \%$, por lo que fue imposible calcular de manera confiable la $\mathrm{CL}_{50}$. A pesar de esto, en el caso del extracto de cloruro de metileno (el de mayor mortandad), con la exposición a 100 ppm se alcanzó $70 \%$ de viabilidad en promedio (figura 3). El promedio de viabilidad en los nauplis expuestos al extracto hexánico fue similar al control para los tratamientos de 1, 5, 10 y 30 ppm (90\%). No obstante, los tratados con 100 ppm disminuyeron significativamente $(p<0.05)$ la viabilidad $(75 \%)$ respecto 
al control (Figura 3). Por ello, de acuerdo con la clasificación de toxicidad reportada previamente para crustáceos, anfibios, peces e insectos (Nguta et al. 2012), se puede considerar que el extracto obtenido con cloruro de metileno es ligeramente tóxico.

Finalmente, los ensayos de toxicidad en A. salina de los extractos de jonote mostraron que en el extracto metanólico no se encontraron diferencias estadísticamente significativas ( $P>0.05$ ) en ninguna de las concentraciones probadas (figura 3). El promedio de viabilidad en los controles, sin la adición de ningún extracto, se mantuvo alrededor de $90 \%$, mientras que, con la adición de las diferentes concentraciones, el promedio se mantuvo por encima de $80 \%$ con una variabilidad de $18 \%$ (figura 3 ).

En el caso del extracto obtenido con cloruro de metileno (figura 3), se encontraron diferencias estadísticamente significativas en todas las concentraciones probadas $(p<0.05)$ en comparación con el control. Sin embargo, con ninguna de las concentraciones probadas de los extractos se alcanzó una viabilidad menor o igual a $50 \%$, por lo que fue imposible calcular de manera confiable la CL50. A pesar de esto, en el caso del extracto de cloruro de metileno (el de mayor mortandad), con la exposición a 100 ppm se alcanzó $70 \%$ de viabilidad en promedio (figura 3). El promedio de viabilidad en los nauplis expuestos al extracto hexánico fue similar al control para los tratamientos de 1, 5, 10 y $30 \mathrm{ppm}$ (90\%). No obstante, los tratados con 100 ppm disminuyeron significativamente $(p<0.05)$ la viabilidad $(75 \%)$ respecto al control (figura 3).

Por ello, de acuerdo con la clasificación de toxicidad reportada previamente para crustáceos, anfibios, peces e insectos (Nguta et al. 2012), se puede considerar que el extracto obtenido con cloruro de metileno es ligeramente tóxico.
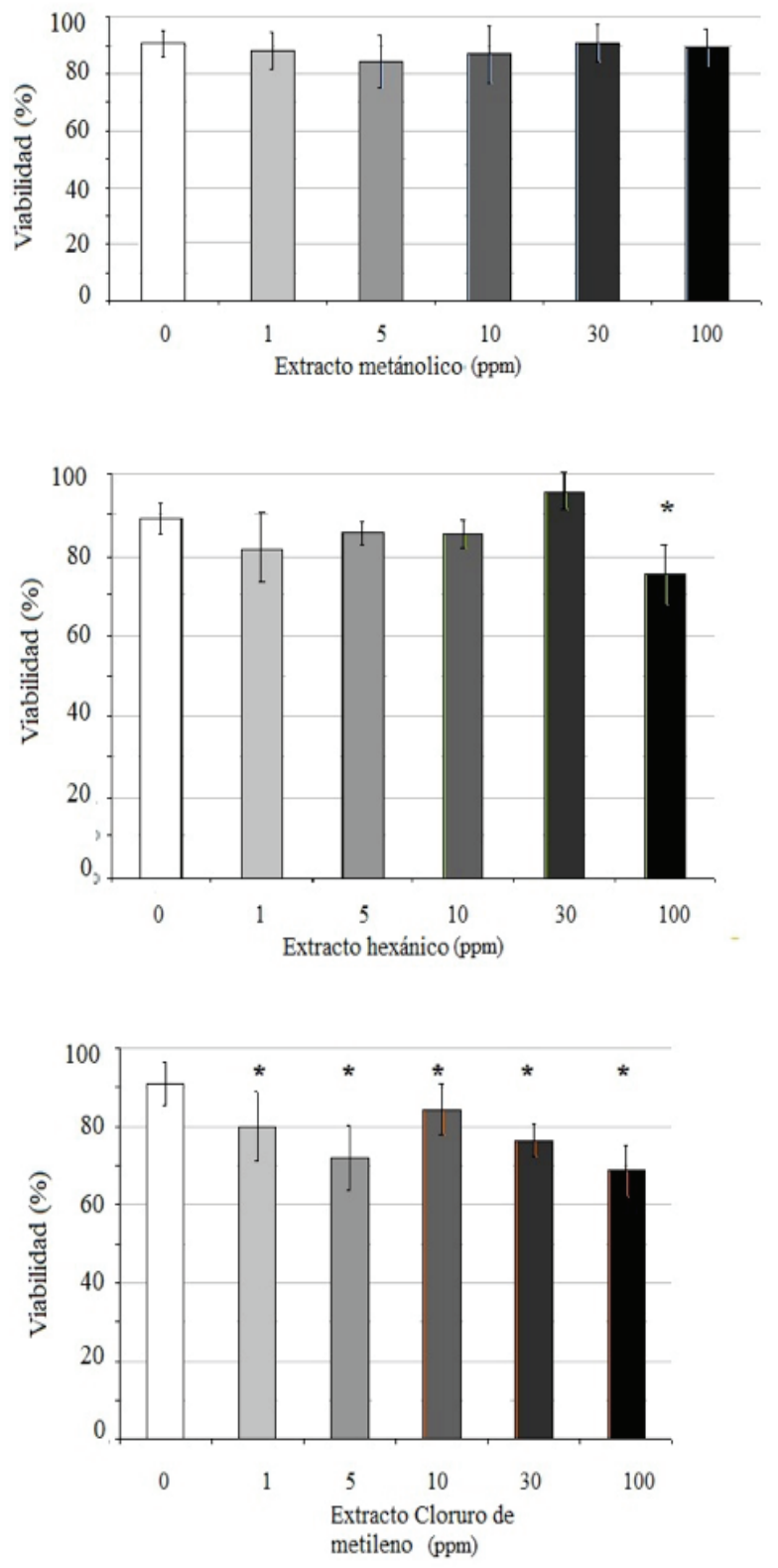

Figura 3. Efecto de diferentes concentraciones de los extractos de $H$. appendiculatus sobre la viabilidad de A. franciscana . *: indica diferencias significativas respecto al control. 


\section{Conclusiones}

De acuerdo con el estudio, los extractos de jonote de diferentes polaridades tienen actividades biológicas diversas. El extracto metanólico de hojas $H$. appendiculatus muestra capacidad antioxidante y el mayor contenido de fenoles totales, además de una toxicidad nula, mientras que el extracto de cloruro de metileno exhibió una actividad tóxica ligera, así como actividad antimicrobiana contra E. coli. Finalmente, el extracto hexánico no muestra actividad antioxidante ni antimicrobiana, aunque sí mostró actividad tóxica ligera.

\section{Agradecimientos}

Las bacterias Escherichia coli y Bacillus thuringiensis Krustaki fueron amablemente donadas por la Dra. Blanca Barrera Figuera y la Dra. Karin Navarro, respectivamente (UNPA).

\section{LitERATURA CITADA}

Bauer AW, Kirby WMM, Sherris JC, Turck M. 1996. Antibiotic susceptibility testing by standardized single disk method. American Journal Clinical Pathology 45(4): 493-496. https://doi.org/10.1093/ajcp/45.4_ts.493

Cabrera-Carrión JL, Jaramillo-Jaramillo C, Dután-Torres F, Cun-Carrión J, García PA, Rojas de Astudillo L. 2017. Variación del contenido de alcaloides, fenoles, flavonoides y taninos en Moringa oleifera Lam. en función de su edad y altura. Bioagro 29(1): 53-60.

Chinchilla-Carmona M, Valerio-Campos I, Sánchez-Porras R, Bagnarello-Madrigal V, Martínez-Esquivel L, González-Paniagua A, Alpizar-Cordero J, Cordero-Villalobos M, Rodríguez-Chaves D. 2004. Actividad contra Leishmania sp. (Kinetoplastida: Trypanosomatidae) de plantas en una Reserva Biológica de Costa Rica. Revista Biología Tropical 62(3): 1229-1240.

Cunill JM. 2015. Contribución al estudio etnofarmacológico del jonote (Heliocarpus appendiculatus Turcz.) en Jonotla, Puebla, México. Tesis de Licenciatura. Ingeniero en Restauración Forestal. Universidad Autónoma Chapingo.

Escalona-Arranz JC, Pérez-Rosés R, Urdaneta-Laffita I, Morris-Quevedo H, Camacho-Pozo MI, Rodríguez-Amado J, Sierra G. 2018. Role of polyphenols in the antimicrobial activity of ethanol Tamarindus indica L leaves fluid extract. Boletín Latinoamericano y del Caribe de Plantas Medicinales y Aromáticas 12(5): 516-522.

Frei B, Baltisberger, Sticher O, Heinrich M. 1998. Medical ethnobotany of the Zapotecs of the Isthmus-Sierra (Oaxaca, Mexico): Documentation and assessment of indigenous uses. Journal of Ethnopharmacology 62(2): 149-165. https://doi.org/10.1016/S0378-8741(98)00051-8

González AM, Presa MF, Lurá MC. 2003. Ensayo de toxi- cidad a Artemia salina: puesta a punto y aplicación a micotoxinas. Revista FABICIB 7(2): 117-122. https://doi. org/10.14409/fabicib.v7i1.726

Guerra-Ramírez D, Hernández-López A, Guerra-Ramírez P, Reyes-Trejo B, Ibarra-Vega P. 2019. Estudio de la actividad biológica de Heliocarpus appendiculatus Turcz en Artemia franciscana. Congreso Internacional de Productos Naturales de Chapingo, México. Química, Biología y Agronomía. Texcoco de Mora, Estado de México, México.

[INEGI] Instituto Nacional de Estadística y Geografía. [internet]. 2015. Marco Geoestadístico. [cited: 2019 april]. Disponible en: https://www.inegi.org.mx.

Jorge P, Troncoso L. 2016. Capacidad antioxidante del fruto de la Opuntia apurimacensis (ayrampo) y de la Opuntia ficus-indica (tuna). Anales de la Facultad de Medicina 77(2): 105-109. http://doi.org/10.15381/anales. v77i2.11812

Kraujalis P, Venskutonis PR, Kraujalienè V, Pukalskas A. 2013. Antioxidant properties and preliminary evaluation of phytochemical composition of different anatomical parts of amaranth. Plant Foods for Human Nutrition 68(3): 322-328. https://doi.org/10.1007/s11130013-0375-8

Leonti M. 2018. Jonote o Majahua. In: Geck MS, editor. Medicina tradicional y herbolaria zoque. San Cristóbal de las Casas, Taller Editorial La Cosecha, pp. 26-27.

Macía MJ. 1999. Plantas de fibra del género Heliocarpus (Tiliaceae) en la Sierra Norte de Puebla, México. Candollea 54(1): 113-123.

Mendoza-Jiménez YL, Eusebio-Moreno JC, Álvarez-García R, Abreu-Corona A, Vargas-Hernández G, Téllez-Jurado A, Tovar-Jiménez X. 2018. Actividad antioxidante de los hidrolizados proteicos del frijol común (Phaseolus vulgaris) cv negro primavera-28 y flor de Durazno. Biotecnia 20(2): 25-30.

Nguta JM, Mbaria JM, Gakuya DW, Gathumbi PK, Kabasa JD, Kiama SG. 2013. Evaluation of acute toxicity of crude plant extracts from Kenyan Biodiversity using brine shrimp, Artemia salina L. (Artemiidae). The Open Conference Proceedings Journal 3(1): 30-34.

Patiño D. [internet]. 2018. Diseño e innovación para mejorar artesanías de jonote. [cited 2018 Ago 16] Disponible en: www.conacytprensa.mx/index.php/tecnologia/materiales/23120-diseno-e-innovacion-para-mejorar-trabajo-de-artesanos.

Pennington TD, Sarukhán J. 2005. Árboles Tropicales de México, manual para la identificación de las principales especies. Distrito Federal, México: Fondo de Cultura Económica-Universidad Nacional Autónoma de México.

Quiñones-Gálvez J, Trujillo R, Capdesuñer Y, Quirós Y, Hernández M. 2013. Potencial de actividad antioxidante de extractos fenólicos de Theobroma cacao L. (cacao). Revista Cubana de Plantas Medicinales 18(2): 201-215.

Re R, Pellegrini N, Proteggente A, Pannala A, Yang M, Rice-Evans C. 1999. Antioxidant activity applying an improved ABTS radical cation decolorization assay. Free 
Radical Biology Medicine 26(9-10): 1231-1237. https:// doi.org/10.1016/S0891-5849(98)00315-3

Ramírez-Rojo MI, Vargas-Sánchez RD, Hernández-Martínez J, Martínez-Benavides E, Sánchez-Escalante JJ, Torrescano-Urrutia GR, Sánchez-Escalante A. 2019. Actividad antioxidante de hoja de mezquite (Prosopis velutina). Biotecnia 21(1): 113-119.

Rasband WS. [Internet] 1997-2018. Image J. Bethesda, Maryland, USA: National Institutes of Health. Disponible en: https://imagej.nih.gov/ij/.

Ruelas-Hernández PG, Aguilar-Castillo JA, Sumaya-Martínez MT, López-Guzmán GG, Juárez-Rosete CR, Valdivia-Bernal R, Sánchez-Herrera ML. 2014. Aptitud combinatoria de la actividad antioxidante de extractos de cálices de jamaica. Revista Bio Ciencias 3(1): 28-38. http://dx.doi.org/10.15741/revbio.03.01.04

Roldán-Sabino C, Viñas-Bravo O, Parra-Reyes A, Galero-Martínez NX, Avila-Alejandre AX, Hernández-López A. 2018. Effect of sonication and pressing on recovery of compounds with antioxidant activity from leaves and bark of Byrsonima crassifolia. Journal of Bioengineering and Biomedicine Research 2(3): 1-8.

Sharma OP, Bath TJ. 2009. DPPH antioxidant assay revisited. Food Chemistry 113(4): 1202-1205. https://doi.org/10.1016/j.foodchem.2008.08.008

Standley PC, Steyermark JA. 1949. Flora of Guatemala, Sabiaceae. Fieldiana 24(6): 273-275.

Stevens PF. [internet]. 2001. Angiosperm Phylogeny Web- site. Versión 14. [cited 2017 jul]. Disponible en: http:// www.mobot.org/MOBOT/research/APweb/.

Svetaz L, Zuljan F, Derita M, Petenatti E, Tamayo G, Cáceres A, Cechinel Filho A, Giménez A, Pinzón R, Zacchino SA, Gupta M. 2010. Value of the ethnomedical information for the discovery of plants with antifungal properties. A survey among seven Latin American countries. Journal of Ethnopharmacology 127(1): 137-158. https:// doi.org/10.1016/j.jep.2009.09.034

Valadeau C, Castillo JA, Sauvain M, Lores AF, Bourdy G. 2010. The rainbow hurts my skin: medicinal concepts and plants uses among the Yanesha (Amuesha), an Amazonian Peruvian ethnic group. Journal of Ethnopharmacology 127(1): 175-192. https://doi.org/10.1016/j.jep.2009.09.024

Vibrans H. [internet]. 2009. Malezas de México, Ficha Heliocarpus appendiculatus Turcz. Jonote. [cited 2019 apr 5]. Disponible en: http://www.conabio.gob.mx/malezasdemexico/tiliaceae/heliocarpus/appendiculatus/ficha/ ficha.htm.

Zou Y-F, Ho GTT, Malterud KE, Le NHT, Inngjerdingen KT, Barsett H, Diallo D, Michaelsen TE, Paulsen BS. 2014. Enzyme inhibition, antioxidant and immunomodulatory activities, and brine shrimp toxicity of extracts from the root bark, stem, bark and leaves of Terminalia macroptera. Journal of Ethnopharmacology 155(2): 12191226. 